\title{
Synthesis of super-dense phase of aluminum under extreme pressure and temperature conditions created by femtosecond laser pulses in sapphire
}

\author{
$\operatorname{Vygantas~Mizeikis~}^{a}$, Arturas Vailionis $^{b, c}$, Eugene G. Gamaly ${ }^{d}$, Wenge Yang ${ }^{e}$, Andrei Rode $^{d}$, \\ and Saulius Juodkazis $f$ \\ ${ }^{a}$ Division of Global Research Leaders and Research Institute of Electronics, Shizuoka \\ University, 3-5-1 Johoku, Naka-ku, Hamamatsu 432-8561, Japan \\ ${ }^{b}$ Geballe Laboratory for Advanced Materials, McCullough Bldg. 227, 476 Lomita Mall, \\ Stanford University, Stanford, CA 94305, USA \\ ${ }^{c}$ Stanford Institute for Materials and Energy Sciences, SLAC National Accelerator Laboratory, \\ 2575 Sand Hill Road, Menlo Park, California 94025, USA \\ ${ }^{d}$ Laser Physics Centre, Research School of Physics and Engineering, The Australian National \\ University, Canberra ACT 0200 Australia \\ ${ }^{e}$ HPSynC - Carnegie Institution of Washington, Argonne National Laboratory, 9700 S. Cass \\ Avenue, Argonne IL 60439, USA \\ ${ }^{f}$ Centre for Micro-Photonics, Faculty of Engineering and Industrial Sciences, Swinburne \\ University of Technology, Hawthorn, VIC, 3122, Australia
}

\begin{abstract}
We describe synthesis of a new super-dense phase of aluminum under extreme pressure and temperature conditions created by laser-induced microexplosions in sapphire. Micro explosions in sub-micrometer sized regions of sapphire were induced by tightly-focused femtosecond laser pulses with a temporal length of $\sim 100$ fs and an energy of $\sim 100$ nJ. Fast, explosive expansion of photogenerated high-density plasma created strong heating and pressure transients with peak temperature and pressure of $\sim 10^{5} \mathrm{~K}$ and 10 TPa, respectively. Partial decomposition of sapphire in the shock-compressed sapphire led to formation of nanocrystalline bcc-Al phase, which is different from ambient fcc-Al phase, and was permanently preserved by fast quenching. The existence of super-dense bcc-Al phase was confirmed using X-ray diffraction technique. This is the first observation of bcc-Al phase, which so far has been only predicted theoretically, and a demonstration that laser-induced micro explosions technique enables simple, safe and cost-efficient access to extreme pressures and temperatures without the tediousness typical to traditional techniques that use diamond anvil cells, gas guns, explosives, or megajoule-class lasers.
\end{abstract}

Keywords: laser-induced micro explosions, extreme temperature and pressure, super-dense aluminum

\section{INTRODUCTION}

Under extreme pressure and temperature conditions, such as those existing inside large planets and stars, or under impact by meteorites or powerful explosions, common materials form new dense phases. Due to pressure, these new phases may have compacted atomic arrangement with significantly reduced lattice period or even a completely different symmetry of the crystalline unit cell. Consequently, density of the compressed phases becomes increased, and their physical properties become modified due to stronger overlap between wave functions of neighboring atoms. Compacting of the atomic arrangement under extreme conditions is illustrated schematically in Fig. 1(a). Compressed matter determines processes in the deep Earth as well as in the cores of massive planets. $^{1,2}$ Study of these processes has fundamental importance for interpretation of astrophysical and geophysical data. Also, synthesis of compressed phases has practical significance, since it may provide new classes

Send correspondence to V.M.: dvmzks@ipc.shizuoka.ac.jp

Advanced Fabrication Technologies for Micro/Nano Optics and Photonics V, edited by Winston V. Schoenfeld, Raymond C. Rumpf, Georg von Freymann, Proc. of SPIE Vol. 8249, 82490A - (C) 2012 SPIE CCC code: $0277-786 X / 12 / \$ 18 \cdot$ doi: $10.1117 / 12.906946$ 

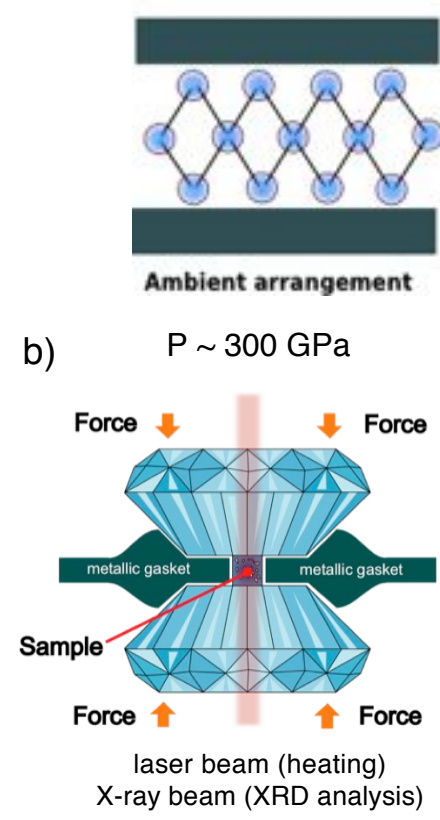

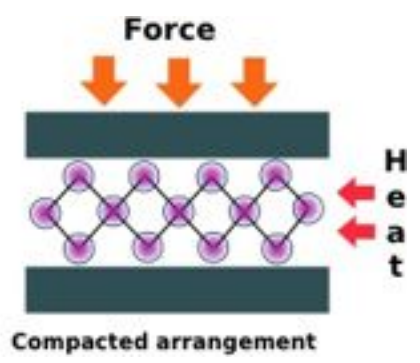

c)

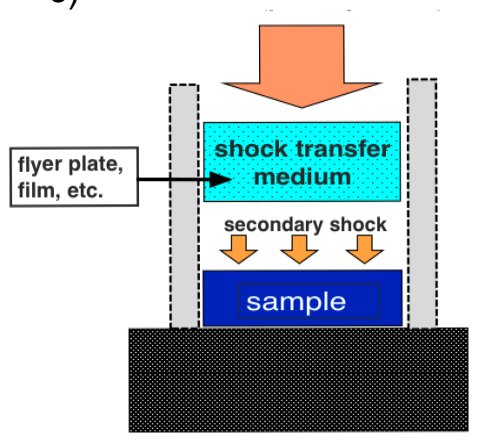

Figure 1. (a) Compacting atomic arrangement of materials under action of external pressure and heating, practical methods of materials compression and their obtainable levels of pressure using (b) diamond anvil cell and (c) shock-wave compression.

of technologically important materials, such as super-hard materials. ${ }^{3-5}$ Here, we describe simple, accessible and cost efficient technique to generate TPa shock wave pressure and $10^{5} \mathrm{~K}$ temperature conditions in transparent solids using micro explosions ignited by tightly focused femtosecond pulses, and report profound modification of sapphire $\left(\mathrm{Al}_{2} \mathrm{O}_{3}\right)$ using this technique. Inspection of modified sapphire regions using X-ray diffraction (XRD) technique indicates formation of nano crystals of metallic aluminum with body-centered cubic (bcc) lattice, which is different from the face-centered cubic (fcc) lattice of metallic aluminum forming under ambient conditions. The high pressure bcc-Al phase was predicted theoretically some time ago, ${ }^{3,6,7}$ but has not been demonstrated till recently, using laser-induced micro explosions technique. ${ }^{8}$ This result demonstrates that extreme conditions generated by laser-induced micro explosions lead to partial decomposition of sapphire into aluminum and oxygen rich regions, and to compression of aluminum-rich regions into nanocrystals of bcc-Al. Also, it validates the previous theoretical predictions and demonstrates a relatively simple and inexpensive route for synthesis of super-dense materials in table-top laser experiments.

\section{EXPERIMENTAL TECHNIQUES FOR HIGH-PRESSURE HIGH-TEMPERATURE MODIFICATION OF MATERIALS}

The quest to create the extreme conditions and to study behavior of materials in laboratory has a long history, ${ }^{9}$ with a dramatic advance in this direction being made by the invention of diamond anvil cell (DAC). ${ }^{10}$ Compression in DAC is achieved by compressing the target material between two pieces of super-hard diamond using external static force, as is shown schematically in Fig. 1(b). Broadband optical transparency of diamond allows easy in situ observation and application of external laser beam for heating, or X-ray beam for structural analysis. However, maximum pressure achievable in static DAC are limited to approximately $300 \mathrm{GPa}$. Even 

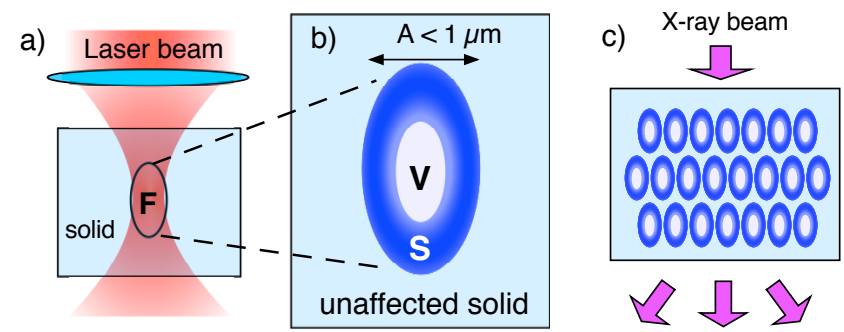

Figure 2. (a) Focusing femtosecond laser pulse inside transparent solid dielectric results in high concentration of optical energy in the focal volume $F$ which exceeds the optical breakdown threshold and ignites micro explosion. (b) Hot electronion plasma photo excited by the laser pulse expands explosively, generating a shock wave and compressing the hot material against colder unaffected solid, and creating a voxel consisting of void $V$ surrounded by a shell $S$ of densified material. Voxel is permanently preserved by fast cooling. (c) Processed area of the material containing arrays of voxels is inspected by XRD technique in order to detect the compressed phases of material in the shells of voxels.

higher pressure/temperature conditions have been created by dynamic shock wave compression. In this method, mechanical energy derived from a powerful primary source, such as gas gun, detonation of explosives, or ablative action of kilo- or megajoule-class laser pulses ${ }^{11-15}$ acts on a transfer medium, for example metallic plate, which subsequently impacts on the sample and transfers its kinetic energy, thus generating shock wave. Implementation of such transient compression, illustrated schematically in Fig. 1(c), allows generation of TPa pressure. Combination of static and dynamic compression (i.e., shock compresion of statically pre-compressed samples) can be also used to reach even higher pressures. ${ }^{15}$ During the last century, pressure and temperature records have increased continuously, and now it is possible to obtain multimegabar static pressures, with simultaneous heating by laser up to $3000 \mathrm{~K} \cdot{ }^{1,16-18}$ However, classical methods of compression typically require facilities that are complex, expensive, and sometimes dangerous to operate, especially in the case of explosions.

Fortunately, recent research has indicated a safer, easier, and cheaper method to create extreme pressure and temperature conditions. This method does not require mechanical source of force, but is based purely on the interaction of ultrafast laser pulses with solid, and can be realized using safe and simple table-top laser experiments. The underlying principle of this technique is based on the circumstance that powerful explosion ignited in the bulk of solid can strongly shock and the surrounding area. For macro-scale explosions, this fact is best evidenced by compressed material found in the sites of underground nuclear detonation. ${ }^{19}$ First evidence of similar effects occurring due to micro-scale explosions ignited by tightly focused femtosecond laser pulses inside fused silica, quartz, sapphire, and other transparent materials was obtained some time ago. ${ }^{20}$ Theoretical analysis of the processes accompanying femtosecond dielectric breakdown in solids has indicated direct analogy between macroscopic and microscopic explosions. ${ }^{21}$ Tightly focused low-energy femtosecond laser pulse can generate explosions having accompanying shock-wave pressure and heat transients with amplitudes $\left(10^{14} \mathrm{~Pa}\right.$ and $10^{5} \mathrm{~K}$, respectively) comparable to those of macro-explosions, in particular nuclear explosions. It is thus possible to modify sub-micrometric volumes in various materials by extreme pressure and temperature conditions using pulses of a low-energy (microjoule class) femtosecond laser. For comparison, generation of similar conditions in a macroscopic region of sample using shock wave originating from ablative action of a nanosecond laser using the scheme depicted in Fig. 1(c) would require kilo- or mega-joule class laser system.

Schematic explanation of these effects is shown in Fig. 2. Under tight focusing, typically using oil-immersion lens with numerical aperture NA $>1$, intensity of the laser pulse at the focus approaches $10^{21} \mathrm{~W} / \mathrm{m}^{2}$ and exceeds optical breakdown threshold (Fig. 2(a)). Optical energy density deposited in the sub-micron volume of material due to impact ionization and non-linear multi-photon absorption is on the order of several $\mathrm{MJ} / \mathrm{cm}^{3}$, several times higher than the strength of any material. This causes the solid in the focal region to superheat and form a plasma. The confined plasma explodes and generates a powerful shock wave that expands out of the focal volume and compresses the hot material against pristine hard surroundings. ${ }^{20-22}$ Shock wave pressure of expanding plasma can reach over 100 Megabar $(1 \mathrm{TPa})$, triggering dramatic changes in the material properties. The effect of shock wave (Fig. 2(b)) is formation of sub-micron laser-modified voxel (volume element) consisting of a void at the 


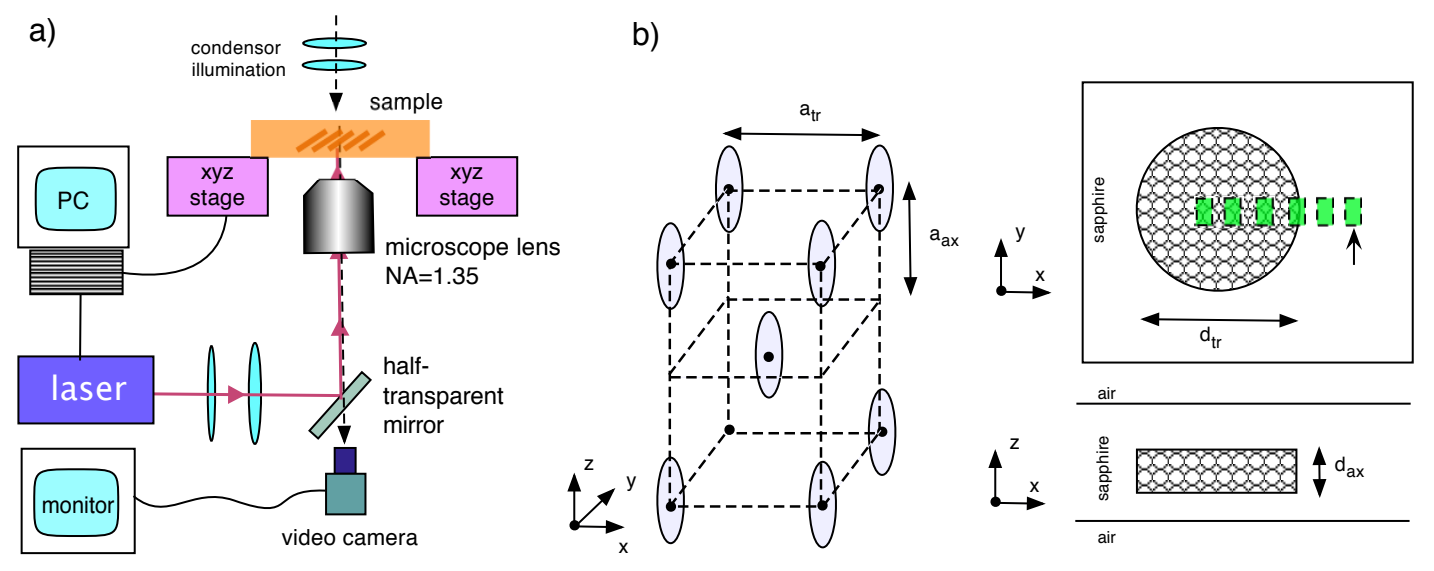

Figure 3. (a) Optical setup for photo modification of materials using fs laser ignited micro explosions.(b) Layout of laserprocessed area, and arrangement of voxels within it. Series of shaded squares indicate locations probed by X-ray beam during XRD characterization.

center of the focal region, and a surrounding shell consisting of densified material due to the material ejected by micro explosion from the void. The modified region cools down to the ambient temperature within about $1 \mathrm{~ns}$ after the laser pulse, thus quickly freezing the compressed shell. This preserves compressed phases for subsequent inspection by XRD or other non-invasive methods as shown in Fig. 2(c).

\section{APPLICATION OF FEMTOSECOND LASER-INDUCED MICRO EXPLOSIONS TECHNIQUE IN SAPPHIRE}

In his section we will describe practical realization of the laser micro explosions technique for modification of sapphire, and provide crucial estimates of laser irradiation conditions and resulting pressure and temperature transients.

\subsection{Practical realization and experimental details}

Optical setup used to modify sapphire in our experiments is shown schematically in Fig. 3(a). The laser source was a Hurricane X (Spectra Physics) regeneratively-amplified Ti:Sapphire femtosecond laser system with a central wavelength of $800 \mathrm{~nm}$, a pulse temporal length of about $150 \mathrm{fs}$, and a repetition rate up to $1 \mathrm{kHz}$. The laser pulses were strongly attenuated to the required pulse energy using variable neutral density filter, and focused in the sample using an oil-immersion objective (Olympus) with numerical aperture $N A=1.4$. At the focus the pulse energy was $135 \pm 6 \mathrm{~nJ}$, and a corresponding peak intensity was $I_{0}=487 \mathrm{TW} / \mathrm{cm}^{2}$. The sapphire sample was a $80 \mu \mathrm{m}$-thick polished slab (Shinkosha Co., Ltd.), and was patterned by extended 3D ordered arrays of closelyspaced voxels in order to maximize the amount of modified material and to allow easier inspection of the laserprocessed areas by XRD. The processed area is shown schematically in Fig. 3(b). For patterning, the sample was mounted on a high-precision three-dimensional translation stage controlled by piezoelectric transducers (Physik Instrumente), and translated along a pre-defined 3D trajectory between consecutive laser shots. The processed volume of sapphire was disk-shaped, with surfaces of the disk parallel to the slab. Such shape was chosen to suppress generation of cracks, which tend to develop near the corners of rectangular patterned regions. Diameter of the processed area in the lateral direction was $d_{t r}=50 \mu \mathrm{m}$, and its thickness in the axial (beam focusing) direction was $d_{a x}=70 \mu \mathrm{m}$. In this area voxels were arranged into 3D arrays with bcc symmetry, and a lattice period of $a_{t r}=2 \mu \mathrm{m}$ along the transverse direction $(x-y$ plane). Along the longitudinal (beam focusing, or $z$-axis) direction, spacing between neighboring planes of voxels was $a_{a x}=2 \mu \mathrm{m}$, but adjacent planes were laterally shifted by half of the lattice period in order to prevent overlap between neighboring layers of voxels. Top layer of the processed area was about $40 \mu \mathrm{m}$ under the surface of sapphire slab. To compensate for the loss of 
tightness of focusing due to aberrations and achieve uniform patterning of the sample at various focusing depths, pulse energy was gradually increased with the focusing depth during the patterning. The laser processing was monitored in situ using a video camera and a monitor. Voids inside sapphire are well distinguishable by their optical contrast. To detect new phases in the laser-processed areas, series of XRD measurements were conducted at various locations of the sample, starting outside the processed area for reference, and gradually moving towards the center of processed area as indicated by shaded squares in Fig. 3(b). Details of XRD characterization will be given later.

\subsection{Estimates of experimental parameters}

For the estimates of our experimental conditions, physical concepts and results described in our earlier works ${ }^{21,22}$ are used in the following Sections.

\subsubsection{Maximum pressure and temperature}

Under excitation by femtosecond laser pulses with intensity $\sim 10^{14} \mathrm{~W} / \mathrm{cm}^{2}$, combined action of avalanche and multi-photon ionization processes converts sapphire into plasma at the beginning of the laser pulse. The average ionization rate of $\sim 10^{15} \mathrm{~s}^{-1}$ during the pulse results in stripping up to 4-5 electrons from $\mathrm{Al}$ and $\mathrm{O}$ atoms, and creating plasma with an electron density of up to $\sim 10^{23} \mathrm{~cm}^{-3}$. At density well above the critical electron density of $1.7 \times 10^{21} \mathrm{~cm}^{-3}$ at the $800 \mathrm{~nm}$ wavelength, ionization and recombination rates become comparable at the very beginning of the pulse, and thus the Saha ionization equilibrium is established in the plasma. Optical properties of the ionized sapphire are drastically different from those of crystalline sapphire: increased absorption and decreased absorption depth lead to an increase in the absorbed energy density. The electron-ion scattering rate $\nu_{e i}$ is on the order of the plasma frequency $\omega_{p e}: \nu_{e i} \approx \omega_{p e} \approx 10^{16} \mathrm{~s}^{-1}$, and the real and imaginary parts of the refractive index are $n \approx k \approx \sqrt{\omega_{\text {pe }} / 2 \omega_{\text {las }}} \approx 2$ under these conditions. The absorption length $l_{\text {abs }}=c /\left(\omega_{\text {las }} k\right)$ decreases down to $\sim 64 \mathrm{~nm}$, while the absorption coefficient $A=4 n\left[(n+1)^{2}+n^{2}\right]^{-1}$ increases to $\sim 0.62$. The estimated absorbed energy density reaches $\sim 3 \mathrm{MJ} / \mathrm{cm}^{3}$ which corresponds to the maximum electron temperature in the absorbing volume of $10-50 \mathrm{eV}$ and maximum pressure of $\sim 10 \mathrm{TPa}$, much larger than the bulk modulus of sapphire. ${ }^{23}$ Graphical summary of physical processes taking place during the laser pulse is given in Fig. 4(a).

\subsubsection{Generation of shock wave and formation of voxel}

Energy of the laser pulse is efficiently deposited into electrons, converting the sapphire into a mixture of $\mathrm{Al}$ and $\mathrm{O}$ ions in the see of hot electrons. Hydrodynamic motion begins after this conversion is complete. The super-heated solid density plasma then generates a powerful shock wave, which expands from the focal volume and compresses material against the cold bulk solid into a shell with increased density. Simultaneously, rarefaction wave decreases the mass density behind the front of the shock wave, i.e., at the geometrical center of the focal region. Size of the void and the compressed shell directly relates the maximum pressure and the absorbed energy to the modulus of sapphire crystal through the laws of mass and energy conservation. These processes are outlined graphically in Fig. 4(b).

Sapphire is one of the hardest natural materials, with the average Young modulus in the range of $Y_{s}=$ $3 B(1-2 \sigma) \approx 400 \mathrm{GPa}$, here, $\sigma \simeq 0.25$ is the Poisson's ratio. ${ }^{23}$ Energy dissipated by the shock wave, $E_{s w}=$ $4 Y_{s} r_{s w}^{3} / 3<E_{a b s}$ is the lowermost estimate for the absorbed pulse energy $E_{a b s}$ (it is also utilized for ionization, heating and compression). Energy and mass conservation laws dictate that volume in which energy is absorbed can not be larger than that the void volume. Thus, a very conservative estimate for the maximum pressure is $P_{\text {max }} \geq E_{a b s} / V_{\text {void }}=Y_{s}\left(r_{s w} / r_{\text {void }}\right)^{3}$. This estimate can be done using experimentally measured quantities. Taking the relation between the measured void size and shock-affected area $r_{s w} \cong 2 r_{\text {void }}{ }^{21,22}$ the maximum pressure should be at least $P_{\max } \geq 8 Y_{s} \approx 3 \mathrm{TPa}$.

The size of the voids and the compressed shell thickness were measured using transmission and scanning electron microscopies (TEM \& SEM) after opening the enclosed laser-affected zone by focussed ion-beam milling. The average radius of the combined void and shell structures was $\sim 360 \mathrm{~nm}$, twice as large as the shell thickness of $\sim 180 \mathrm{~nm}$. Assuming conservation of mass, the average shell density was $\delta=1.14 \rho_{s}=4.54 \mathrm{~g} / \mathrm{cm}^{3}$, where $\rho_{s}=3.98 \mathrm{~g} / \mathrm{cm}^{3}$ is the initial density of sapphire. This shell was identified as amorphous material by TEM and chemical etching since amorphous sapphire has a much higher solubility in hydrofluoric acid compared 
a)
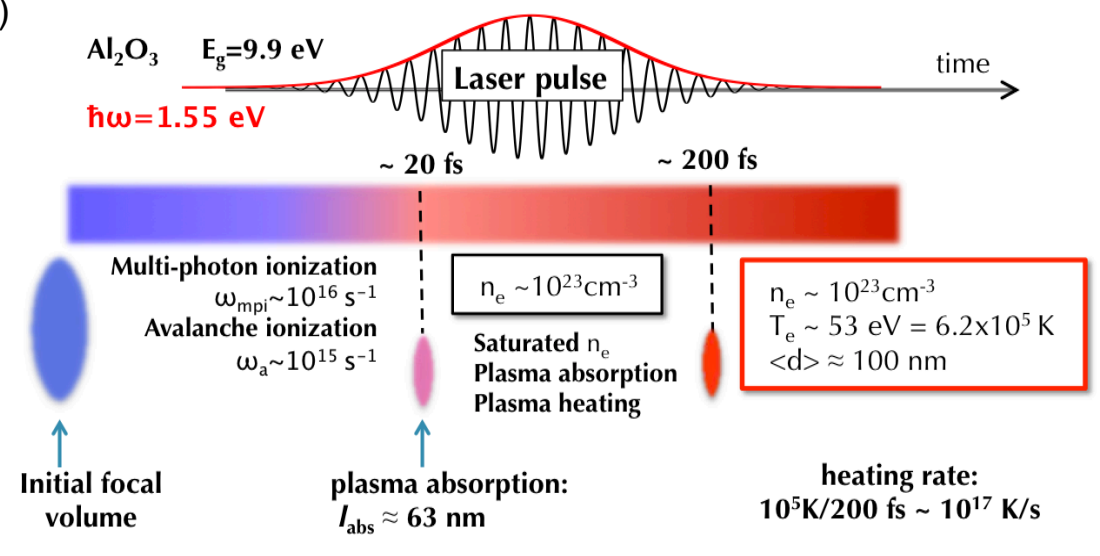

b)

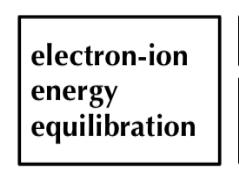

Energy density:

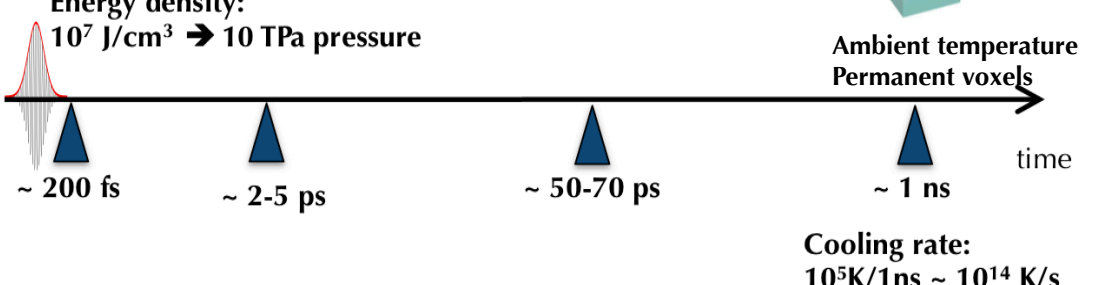

Figure 4. Timeline of physical processes in photo-excited sapphire, (a) during the action of laser pulse, (b) after the excitation till full relaxation.

with crystalline sapphire. ${ }^{22}$ Taking the measured radius of the shock-modified volume $r_{s w} \approx 360 \mathrm{~nm}$, void radius $r_{\text {void }} \approx 180 \mathrm{~nm}$, and $Y_{s}=300 \mathrm{GPa}$, we estimate the upper limit for the absorbed laser energy, $E_{a b s} \geq$ $Y_{s} \times 4 \pi r_{s w}^{3} / 3=59 \mathrm{~nJ}$. Considering the ionization losses, this corresponds to the maximum electron temperature of $T_{e}^{\max }=44 \mathrm{eV}$.

\subsubsection{Dynamics of excitation and relaxation processes}

Temporal evolution of the physical processes described in the previous Sections is summarized in Fig. 4. We stress here extremely fast rate of material heating, of $\sim 10^{17} \mathrm{~K} / \mathrm{s}$ achievable using our experimental method. For comparison, shock compression and decompression of materials under the action of shock waves induced by explosives provides much lower heating rates of $\sim 10^{12} \mathrm{~K} / \mathrm{ns}$. Fast cooling of the hot photo-excited material is another advantage of this technique: ambient temperature is reached within approximately $1 \mathrm{~ns}$ after the excitation, yielding the cooling rate of $10^{14} \mathrm{~K} / \mathrm{s}$. Due to such fast cooling, the compressed shell becomes quenched in strongly compressed state, thus helping to preserve the new compressed phases for subsequent inspection.

\section{INVESTIGATION OF LASER-PROCESSED SAPPHIRE BY XRD TECHNIQUE}

XRD characterization of the laser-processed regions was carried by angle-dispersive X-ray micro-diffraction at HPCAT beamline 16-BM-D of the Advanced Photon Source, Argonne National Laboratory. The monochromatic X-ray beam with energy of $33 \mathrm{keV}(\lambda=0.37571 \AA$ ) produced by a Si (111) monochromator was focused by a pair of Kirkpatrick-Baez $(\mathrm{K}-\mathrm{B})$ mirrors to an area of $(5.0 \times 10.0) \mu \mathrm{m}^{2}$ (width $\times$ height) estimated at FWHM. X-ray 
a)

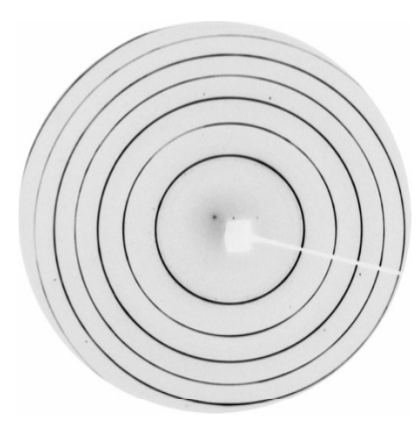

b)

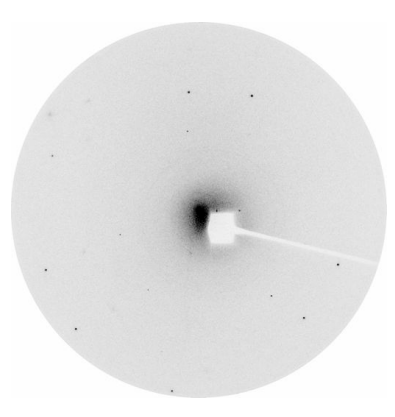

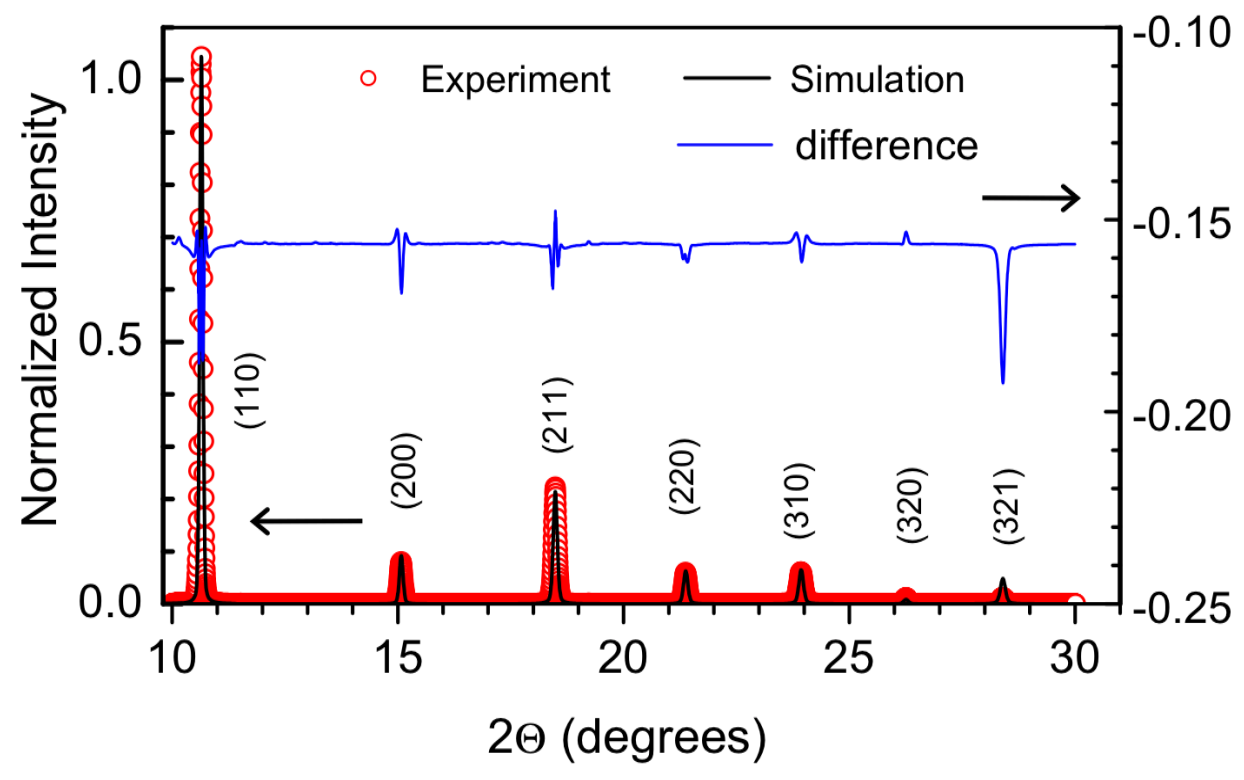

Figure 5. Raw XRD patterns (a) in laser-processed area, (b) in pristine sapphire, (c) comparison between azimuthally integrated XRD spectra: experimental data (dots) and simulation assuming bcc-Al structure (solid line). Difference between the experiment and simulation is also indicated by solid line.

diffraction patterns were collected in the transmission mode using a Mar345 Image Plate with typical exposure time per image of 50-100 s. The sample-to-detector distance and detector tilts were calibrated with a $\mathrm{CeO}_{2}$ (NIST) standard. All two-dimensional XRD images were integrated to one-dimensional diffraction patterns using FIT2D software. XRD pattern analysis, structure determination and Rietveld refinement was performed using X'Pert HighScore Plus (PANalytical Inc.) and Materials Studio (Accelrys, Inc.) software. Theoretical powder X-ray diffraction patterns expected for fcc-Al, bcc-Al, sapphire and high pressure $\mathrm{AlO}_{x}$ phases were calculated using the Materials Studio package (Accelrys, Inc.).

Figure 5(a) and (b) show raw 2D XRD images collected at laser-modified and intact areas in the sample. There are pronounced diffraction rings in the modified regions, while pristine regions show only a weak background. An azimuthally integrated XRD pattern of Fig.5(a) was thoroughly compared with the X-ray diffracted peak positions of known and theoretically predicted high-pressure $\mathrm{Al}_{2} \mathrm{O}_{3}$ phases, ${ }^{24}$ but no satisfactory match was obtained. Further examination revealed that the measured peak positions could be perfectly indexed as a bcc-Al structure (space group $\operatorname{Im} 3 m$ ) with a lattice constant $a=2.864 \AA\left(11.75 \AA^{3} /\right.$ atom), which matches a predicted, ${ }^{3,6,25}$ but not yet experimentally observed, high-pressure $\mathrm{Al}$ phase; this bcc-phase is $41 \%$ more dense than the native fcc phase of Al. Figure 5(c) shows comparison between the experimental XRD pattern 
and Rietveld refined profile of bcc-Al as well as difference between the experimental and calculated curves. The observed R-values of the fit criteria $R_{w p}=0.08$ and $R_{p}=0.05$ confirm the high quality fit for such high symmetry structure. Shapes of the Bragg peaks were fitted using Rietveld refinement with crystallite size and micro-strain as fitting parameters, yielding the crystallite size of $17.9 \mathrm{~nm}$ and strain of $0.26 \%$, closely matching the values calculated using Scherrer algorithm: ${ }^{26} L=K \lambda /(\beta \cos \theta)$, where $L=18 \pm 2 \mathrm{~nm}$ is size of the nanoparticle, $K=0.891$ is a constant, $\beta$ is the FWHM of the diffraction peak, and $\theta$ is the diffraction angle.

To prove that bcc lattice is formed by aluminum atoms only, we have thoroughly examined the possibility of oxygen participation. The indexing of the XRD pattern revealed that the observed Bragg peaks originate from a material having bcc unit cell with a lattice constant $a=2.866 \AA$ and space group Im-3m (229). Such unit cell has only two unique atoms at atomic coordinates of $(0,0,0)$ and $(1 / 2,1 / 2,1 / 2)$. Hence, the two shortest interatomic distances are $2.482 \AA$ (between the corner and center atoms) and $2.866 \AA$ (between the corner atoms). Since micro-explosions were confined in the bulk of crystalline $\mathrm{Al}_{2} \mathrm{O}_{3}$, the only constituents that can contribute to the observed Bragg peaks are $\mathrm{Al}$ and $\mathrm{O}$. The shorter interatomic distance agrees well with double atomic radius of $\mathrm{Al}$ of $1.25 \AA,{ }^{27}$ thus suggesting $\mathrm{Al}-\mathrm{Al}$ bond. Since atomic radius of oxygen is much smaller, $0.6 \AA$, length of Al-O bond would be about $1.85 \AA$. For instance, bonds of similar length can be found in crystalline $\mathrm{Al}_{2} \mathrm{O}_{3}$ lattice, where two shortest interatomic distances are $1.852 \AA$ and $1.975 \AA .{ }^{32}$ In our bcc lattice, the presence of Al-O bond would result in a XRD pattern drastically different from the observed one. Hence, oxygen cannot form bonds with aluminum in the identified bcc structure with $a=2.866 \AA$, and does not contribute to the experimental XRD pattern shown in Figure 5(c). Consequently, both atoms in the bcc unit cell are Al, and the observed XRD pattern is due to nanocrystallites of pure aluminium. The $\mu \mathrm{XRD}$ provides a non-destructive verification of the new bcc-Al phase which is stabilized in compressed enclosure as estimated by an observed co-existence of bcc- as well as a $\sim 60 \mathrm{GPa}$-compressed fcc-Al phase. ${ }^{8}$ Thus, our XRD data clearly indicates formation of bcc-Al nano crystallites in laser processed sapphire due to extreme pressure and temperature conditions.

High-pressure structural modification of crystalline metallic Al phases has been the subject of first-principles theoretical studies, ${ }^{3,6,25}$ because ambient fcc-Al is regarded as a model material for testing structural phase stability of simple metals, and is usable as a pressure standard in the megabar range. ${ }^{5,28}$ The theories predict a sequence of phase transitions from the native fcc-Al phase to hexagonal closely-packed (hcp) phase at an estimated static transition pressure within the 120 - $360 \mathrm{GPa}$ range and from hcp to bcc phase within the 200 - $560 \mathrm{GPa}$ range, or a direct fcc to bcc transition at the estimated pressure of $272-340$ GPa. At even higher, terapascal pressures, host-guest structures have been predicted. ${ }^{29}$ Despite the use of state-of-the-art compression techniques, only the existence of hcp-Al phase has been confirmed experimentally so far. ${ }^{5,30}$

\section{PHYSICAL PROCESSES LEADING TO FORMATION OF BODY-CENTERED ALUMINUM}

Formation of crystalline aluminum in aluminium oxide is experimental evidence of a quite unusual and unexpected phenomenon, namely, spatial separation of oxygen and aluminum ions in conditions of complete confinement and preserved stoichiometry. This section presents qualitative explanation for this effect.

The ultrashort laser pulses with intensity above the optical breakdown threshold breaks bonds of $\mathrm{Al}_{2} \mathrm{O}_{3}$ and ionize constituent atoms into plasma which exists until the material cools down to below the thermal ionization threshold. Whilst this plasma exists, the $\mathrm{Al}$ and $\mathrm{O}$ ions diffuse and scatter at different rates, enabling spatial separation of the Sapphire constituents to occur. This process is depicted qualitatively in Fig. 6 and is discussed below.

In electrically neutral sapphire, spatial separation between $\mathrm{O}$ and $\mathrm{Al}$ atoms due to diffusion would be physically impossible even if atomic bonds were broken due to the elevated temperature, because mean free path of atoms would be shorter than the interatomic spacing, and cross-section for atomic collisions of would be temperatureindependent. Spatial separation via diffusion can only occur in a hot plasma state where atoms are ionized and ionic collisions are governed by the Rutherford scattering with cross-section inversely proportional to the relative energy and reduced mass of the interacting particles. ${ }^{31}$

The electron-ion energy transfer time in plasma is directly proportional to the ion mass: $t_{\text {ei }}^{e n}=M_{\text {ion }}\left(m_{e} \nu_{e i}^{m o m}\right)$; here $\nu_{e i}^{m o m}$ is the electron-phonon momentum exchange rate. Thus, mass difference becomes important at the 


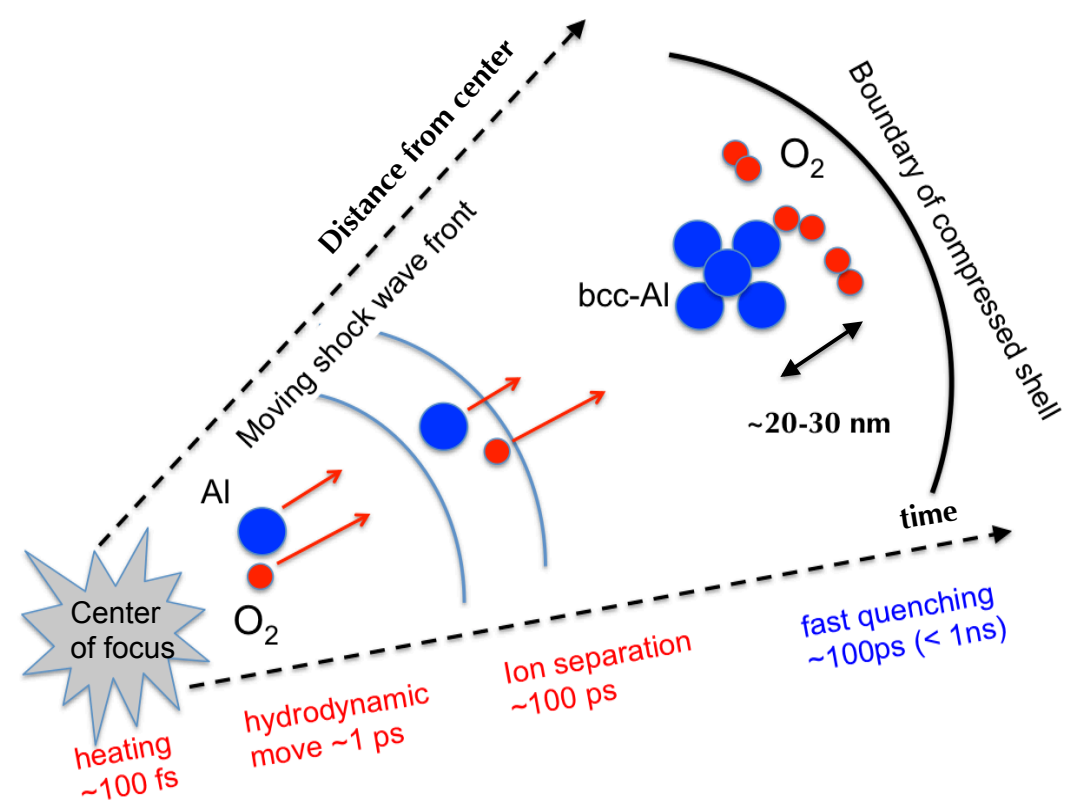

Figure 6. Qualitative picture of separation between $\mathrm{Al}$ and $\mathrm{O}$ ions in hot sapphire.

early stage of plasma formation: lighter O ions are heated stronger, gain more energy, and diffuse faster than heavier $\mathrm{Al}$ ions. As a result, unique non-equilibrium three-temperature condition is established. Difference between the ionic diffusion lengths $\Delta=L_{d}^{O}-L_{d}^{A l}$ indicates spatial separation between the eventual distributions of $\mathrm{Al}$ and $\mathrm{O}$ ions. Diffusion length $L_{d}=\sqrt{D_{d} t_{d}}$ depends on the ion mean free path $l_{m f p}$ and velocity $v_{i o n}$ since $D_{d}=l_{m p f} \nu_{i o n} / 3$. Diffusion continues within time $t_{d}$ till plasma temperature drops below a certain level. Thus, effective spatial separation between $\mathrm{Al}$ and $\mathrm{O}$ ion distributions can be expressed in the following form:

$$
\Delta=L_{d}^{O}\left[1-\left(\frac{D_{A l}}{D_{O}}\right)^{1 / 2}\right]=L_{d}^{O}\left[1-\left(\frac{l_{m f p}^{A l} \nu_{A l}}{l_{m f p}^{O} \nu_{O}}\right)^{1 / 2}\right]
$$

The mean free path, $l_{m f p}=\left(n_{\text {ion }} \sigma_{R}\right)^{-1}$ depends on the Rutherford cross-section $\sigma_{R}=4 \pi e^{4} Z_{1}^{2} Z_{2}^{2} \ln \Lambda \mu^{-1}\left(\nu_{1}-\right.$ $\left.\nu_{2}\right)^{-4}$, which governs Coulomb scattering of ions in plasma; here $Z_{1}$ and $Z_{2}$ are the electrical charges, $\mu=$ $M_{1} M_{2} /\left(M_{1}+M_{2}\right)$ is the reduced mass, and $\left(\nu_{1}-\nu_{2}\right)$ is the relative velocity of interacting particles. At a fixed temperature, differences in the ion velocity, mean free path, and diffusion length are related to different masses of ions. Since ratio between velocities of oxygen and aluminum ions is $\nu_{O} / \nu_{A l}=\left(M_{A l} / M_{O}\right)^{1 / 2}=1.3$, the spatial separation can be expressed through the mass difference as follows:

$$
\Delta=L_{d}^{O}\left[1-\frac{\mu_{A l}}{\mu_{O}}\left(\frac{M_{O}}{M_{A l}}\right)^{5 / 2}\right]=0.33 L_{d}^{O}
$$

we assume here that each ion diffuses in plasma with the average ion mass $M_{a v}=20$ a.u. and $\mu_{A l, O}=$ $M_{A l, O} M_{a v} /\left(M_{A l, O}+M_{a v}\right)$. One can see that spatial separation by tens-of-nm between oxygen and alumnium is possible if diffusion length of oxygen exceeds $100 \mathrm{~nm}$.

The main reason for spatial separation of oxygen and aluminum ions is difference of their diffusion coefficients and the diffusion time $t_{d}$, which define the diffusion length. The Coulomb collision cross section for the temperature range of $10-50 \mathrm{eV}$ only slightly exceeds the atomic cross section and is $\sigma_{R} \approx 10^{-15} \mathrm{~cm}^{2}$. Therefore, 
mean free path in the solid density plasma is comparable to the inter-atomic distance. Plasma lifetime limits the available time during which ion separation can develop. After the laser pulse is gone, thermal ionization by electron impact maintains the ionization process at almost the same level as during the action of laser pulse, because the plasma temperature is much higher than the ionization potential. ${ }^{21}$ Therefore, plasma exists until cooling processes reduce the temperature below the threshold value of $\approx 10 \mathrm{eV}$ due to energy dissipation in the expanding shock wave and heat conduction.

The process of void formation can be interpreted as a rarefaction wave which propagates towards the center of laser-excited volume and maintains high temperature of ions in the central part. The density decrease in the central part of spherical explosion is accompanied by the increase in temperature, keeping the pressure nearly constant in the central region. ${ }^{31}$ The ion mean free path is inversely proportional to the density. According to hydrodynamic simulations of micro-explosions, ${ }^{21}$ mass density at the center is around $0.1 \mathrm{~g} / \mathrm{cm}^{3}$ at $0.9 \mathrm{~ns}$ after the micro-explosion. Hence, mean free path of oxygen ions is within several nanometers, and diffusion rate is $D_{d}^{O} \approx 0.1 \mathrm{~cm}^{2} / \mathrm{s}$. Taking the cooling time $t_{d} \approx 1 \mathrm{~ns}$ in accordance with computer simulations, ${ }^{21}$ the oxygen diffusion length is $L_{d}^{O} \approx 100 \mathrm{~nm}$. Thus, separation of oxygen and aluminum ions in hot and dense plasma due to the difference in the diffusion rates appears to be a viable process responsible for the formation of super-dense bcc-Al crystals.

As mentioned above, detailed analysis of all acquired XRD images has revealed that small fraction of the laser-processed areas probed by XRD also reveal dominant signatures of compressed fcc-Al phase rather than bcc-Al phase. ${ }^{8}$ This can be expected in regions of the material subjected to large pressure, temperature, and mass density variations due to laser exposure. Detailed analysis of these signatures has confirmed fcc lattice symmetry with a lattice constant $a=3.584 \AA$ and the same space group, Fm-3m $(225)^{32}$ as the ambient fcc-Al phase. Atomic volume of the compressed fcc-Al is $V=11.51 \AA^{3}=0.69 V_{0}$, where $V_{0}=16.62 \AA^{3}$ is atomic volume of fcc-Al at ambient conditions. Using the known dependence of atomic volume on the pressure for fcc- $\mathrm{Al},{ }^{5}$ we can estimate that residual pressure that acts on fcc-Al nanocrystallites inside the compressed amorphous $\mathrm{Al}_{2} \mathrm{O}_{3}$ is about $61 \mathrm{GPa}$.

Further insights into formation of the Al nano-crystallites can be obtained by analysis of the known lattice parameters of fcc and bcc Al phases. ${ }^{25}$ Normalizing lattice constant to Bohr radius $(0.529 \AA)$ results in 7.65 Bohr $(a=4.049 \AA)$ for ambient fcc-Al, which is very close to the calculated value of 7.596 Bohr. Assuming that bcc-Al nanocrystallites experience the same residual pressure as compressed fcc-Al, volume reduction ratio for the bcc-Al can be deduced as $V / V_{0}=0.709$. From the atomic volume compression ratios we can calculate the zero-pressure bcc-Al lattice constant of $3.214 \AA$ or 6.073 Bohr. This value is very close to the theoretical prediction for zero-pressure lattice constant of bcc-Al of 6.077 Bohr. ${ }^{25}$ Although local compression might differ in the regions dominated by fcc- and bcc-Al phases, this analysis indicates that nanocrystallites of both phases experience high residual pressure, which might help explain their stability.

The existence of strong residual pressure also indicates that direct analysis of the compressed material which would require to expose voxels to outside environment by mechanical or ion milling, or plasma treatment, cannot be applied due to unavoidable relaxation of the residual stress and irreversible modification of the environment created by laser-induced microexplosions.

\section{CONCLUSIONS}

Successful synthesis of a new stable high-pressure phase of bcc-Al using laser-induced micro explosions illustrates high potential o this simple and highly accessible experimental technique, and confirms previously theoretically predicted transformation of $\mathrm{Al}$ under high pressure. In contrast to the compression of crystalline aluminum using traditional methods, this transformation was achieved in sapphire, without using aluminum as initial material. Separation of ions in hot, laser-excited material, responsible for these findings, may also occur in a wide range of other geophysically and technologically important materials, and may mark a general route of "synthesis via decomposition" that can be exploited in the future. Although amount of new phase material produced by this technique is currently too low for practical purposes, it may be increased using laser systems operating at higher high repetition rates, up to the $\mathrm{MHz}$ range. ${ }^{33}$ 


\section{ACKNOWLEDGMENTS}

This work was supported by the Australian Research Council through the Discovery program. SJ thanks Photon Process lab of Hokkaido University for the use of their laser, Tecdia Ltd. for sample preparation. XRD experiments were performed at HPCAT, which is supported by CIW, CDAC, UNLV and LLNL through funding from DOE-BES, DOE-NNSA and NSF. HPSynC is supported as part of the EFree, an Energy Frontier Research Center funded by DOE-BES under award DE-SC000 1057.

\section{REFERENCES}

1. P. F. McMillan, "New materials from high-pressure experiments," Nature Materials 1, pp. 19-25, 2002.

2. T. Ahrens, "Dynamic compression of Earth materials," Science 207, pp. 1035-1041, 1980.

3. J. A. Moriarty and A. K. McMahan, "High-pressure structural phase transitions in Na, Mg, and Al," Phys. Rev. Lett. 48, pp. 809-812, 1982.

4. P. K. Lam and M. L. Cohen, "Calculation of high-pressure phases of Al," Phys. Rev. B 27, pp. 5986 - 5991, 1983.

5. Y. Akahama, M. Nishimura, K. Kinoshita, H. Kawamura, and Y. Ohishi, "Evidence of a fcc-hcp transition in aluminum at multimegabar pressure," Phys. Rev. Lett. 96, p. 045505, 2006.

6. C. Friedli and N. W. Ashcroft, "Aluminum under high pressure. I. equation of state," Phys. Rev. B 12, pp. 5552-5559, 1975.

7. M. J. Tambe, N. Bonini, and N. Marzari, "Bulk aluminum at high pressure: A first-principles study," Phys. Rev. B 77, p. 172102, 2008.

8. A. Vailionis, E. G. Gamaly, V. Mizeikis, W. Yang, A. V. Rode, and S. Juodkazis, "Evidence of superdense aluminium synthesized by ultrafast microexplosion," Nat. Commun. 2, p. 445, 2011.

9. A. Katrusiak, "High-pressure crystallography," Acta Crystallogr. A64, pp. 135-148, 2008.

10. C. Weir, E. Lippincott, A. V. Valkenburg, and E. Bunting, "Infrared studies in the 1- to 15-micron region to 30.000 atmospheres," J. Res. Natl. Bur. Stand. 63A, pp. 55-62, 1959.

11. H. He, T. Sekine, T. Kobayashi, H. Hirosaki, and I. Suzuki, "Shock-induced phase transition of $\beta-\mathrm{Si}_{3} \mathrm{~N}_{4}$ to c-Si ${ }_{3} \mathrm{~N}_{4}, "$ Phys. Rev. B 76, pp. $11412-11417,2000$.

12. A. Salleo, S. T. Taylor, M. C. Martin, W. R. Panero, R. Jeanloz, T. Sands, and F. Y. Genin, "Laser-driven formation of a high-pressure phase in amorphous silica," Nature Materials 2, pp. 796-800, 2003.

13. R. Cauble, D. W. Phillion, T. J. Hoover, N. C. Holmes, J. D. Kilkenny, and R. W. Lee, "Demonstration of 0.75 Gbar planar shocks in x-ray driven colliding foils," Phys. Rev. Lett. 70, pp. 2102-2105, 1993.

14. D. G. Hicks, P. M. Celliers, G. W. Collins, J. H. Eggert, and S. J. Moon, "Shock-induced transformation of $\mathrm{Al}_{2} \mathrm{O}_{3}$ and LiF into semiconducting liquids," Phys. Rev. Lett. 91, p. 035502, 2003.

15. R. Jeanloz, P. M. Celliers, G. W. Collins, J. H. Eggert, K. K. M. Lee, R. S. McWilliams, S. Brygoo, and P. Loubeyre, "High-pressure geoscience special feature: Achieving high-density states through shock-wave loading of precompressed samples," Proc. Nat. Acad. Sci. 104, pp. 9172-9177, 2007.

16. J. M. Léger, J. Haines, M. Schmidt, J. P. Petitet, A. S. Pereira, and J. A. H. da Jornada, "Discovery of hardest known oxide," Nature 383, p. 401, 1996.

17. A. Zerr, G. Miehe, G. Serghiou, M. Schwarz, E. Kroke, R. Riedel, H. Fueß, P. Kroll, and R. Boehler, "Synthesis of cubic silicon nitride," Nature 400, pp. 340-342, 1999.

18. A. Ruoff, C. Rodriguez, and N. Christensen, "Elastic moduli of tungsten to 15 Mbar, phase transition at 6.5 Mbar and rheology to 6 Mbar," Phys. Rev. B 58, pp. 2998-3002, 1998.

19. N. Greiner, D. Phillips, J. Johnson, and F. Wolk, "Diamonds in detonation soot," Nature 333, pp. 440-442, 1988.

20. E. Glezer and E. Mazur, "Ultrafast-laser driven micro-explosions in transparent materials," Appl. Phys. Lett. 71, pp. 882-884, 1997. 
21. E. G. Gamaly, S. Juodkazis, K. Nishimura, H. Misawa, B. Luther-Davies, L. Hallo, P. Nicolai, and V. T. Tikhonchuk, "Laser-matter interaction in the bulk of a transparent solid: Confined microexplosion and void formation," Phys. Rev. B 73, p. 214101, 2006.

22. S. Juodkazis, K. Nishimura, S. Tanaka, H. Misawa, E. G. Gamaly, B. Luther-Davies, L. Hallo, P. Nicolai, and V. T. Tikhonchuk, "Laser-induced microexplosion confined in the bulk of a sapphire crystal: Evidence of multimegabar pressures," Phys. Rev. Lett. 96, p. 166101, 2006.

23. J. Smyth, S. Jacobsen, and R. Hazen, Comparative crystal chemistry of dense oxide minerals, vol. 41, ch. 6, pp. 157-186. Mineralogical Society of America, Washington, 2000.

24. A. R. Oganov and S. Ono, "The high-pressure phase of alumina and implications for Earth's D" layer," Proc. Nat. Acad. Sci. 102, pp. 10828-10831, 2005.

25. J. C. Boettger and S. B. Trickey, "High-precision calculation of the equation of state and crystallographic phase stability for aluminum," Phys. Rev. B 53, pp. 3007-3012, 1996.

26. P. Scherrer, "Bestimmung der Grösse und der inneren Struktur von Kolloidteilchen mittels Röntgenstrahlen," Nachr. Ges. Wiss. Göttingen 26, pp. 98-100, 1918.

27. J. Slater, "Atomic radii in crystals," J. Chem. Phys. 41, pp. 3199-3204, 1964.

28. D. Batani, A. Morelli, M. Tomasini, A. Benuzzi-Mounaix, F. Philippe, M. Koenig, B. Marchet, I. Masclet, M. Rabec, C. Reverdin, R. Cauble, P. Celliers, G. Collins, L. Da Silva, T. Hall, M. Moret, B. Sacchi, P. Baclet, and B. Cathala, "Equation of state data for iron at pressures beyond 10 Mbar," Phys. Rev. Lett. 88, p. 235502, 2002.

29. C. J. Pickard and R. J. Needs, "Aluminium at terapascal pressures," Nat. Materials 9, pp. 624-627, 2010.

30. N. N. Roy and E. G. Steward, "Compresion of aluminium up to about 200 kbar and evidence of a structure change," Nature 224, p. 905, 1969.

31. L. P. Pitaevskii and E. Lifshitz, Statistical Physics, part 2, vol. 9, Butterworth-Heinemann, 1980.

32. Power diffraction files from Int. Center for Diffraction Data, http://www.icdd.com.

33. T. Südmeyer, S. V. Marchese, S. Hashimoto, C. R. E. Baer, G. Gingras, B. Witzel, and U. Keller, "Femtosecond laser oscillators for high-field science," Nature Photonics 2, pp. 599-604, 2008. 